\title{
NEAR-RINGS OF COMPATIBLE FUNCTIONS
}

\author{
by GÜNTER PILZ \\ (Received 15th September 1978)
}

\section{Summary}

In this paper we study near-rings of functions on $\Omega$-groups which are compatible with all congruence relations. Polynomial functions, for instance, are of this type. We employ the structure theory for near-rings to get results for the theory of compatible and polynomial functions (affine completeness, etc.). For notations and results concerning near-rings see e.g. (10). However, we review briefly some terminology from there. $(N,+,$.$) is a near-ring if (N,+)$ is a group and . is associative and right distributive over + . For instance, $M(A):=\left(A^{A},+, \circ\right)$ is a near-ring for any group $(A,+)$ ( $\circ$ is composition). If $N$ is a near-ring then $N_{0}:=\{n \in N / n 0=0\}$. A group $(\Gamma,+)$ is an $N$-group (we write ${ }_{N} \Gamma$ ) if a "product" $n \gamma$ is defined with $\left(n+n^{\prime}\right) \gamma=n \gamma+n^{\prime} \gamma$ and $\left(n n^{\prime}\right) \gamma=n\left(n^{\prime} \gamma\right)$. Ideals of near-rings and $N$-groups are kernels of $\left(N_{-}\right)$homomorphisms. If $\Gamma$ is a vector-space, $M_{\text {aff }}(\Gamma)$ is the near-ring of all affine transformations on $\Gamma$. $N$ is 2-primitive on ${ }_{N} \Gamma$ if ${ }_{N} \Gamma$ is non-trivial, faithful and without proper $N$ subgroups. The (2-) radical and (2-) semisimplicity are defined similarly to the ring case.

\section{Compatible and polynomial functions}

In (13), an $N$-group ${ }_{N} \Gamma$ ( $N$ a near-ring) is called tame if $N=N_{0}$ and every $N$-subgroup of ${ }_{N} \Gamma$ is an ideal of ${ }_{N} \Gamma$ (= kernel of an $N$-group homomorphism); $N$ is tame if there is some faithful tame $N$-group. We study a similar concept:

Definition 2.1. Let $N$ be a near-ring and ${ }_{N} \Gamma$ an $N$-group.

(a) ${ }_{N} \Gamma$ is gentle if and only if every normal subgroup of $(\Gamma,+)$ is an ideal of ${ }_{N} \Gamma$.

(b) $N$ is gentle if and only if there exists some faithful gentle $N$-group.

We get a lot of interesting examples in the following way.

Definition 2.2 Let $A=(A, \Omega)$ be a (universal) algebra.

(a) $M(A):=\left(A^{A}, \Omega \cup\{\circ\}\right)$, where $\circ$ means the composition of functions; the operations $\omega \in \Omega$ are defined pointwise.

(b) $C(A):=\{f \in M(A) \mid$ for all congruence relations $\equiv$ of $A$ we have that

$$
\forall a, b \in A: a \equiv b \Rightarrow f(a) \equiv f(b)\} .
$$

The functions in $C(A)$ are called compatible.

See (13) for a number of results concerning the structure of compatible near-rings. 
(c) Let $P(A)$ be the subalgebra of $M(A)$ generated by id $_{A}$ and the constant functions. The elements of $P(A)$ are called polynomial functions.

(d) For $n \in N$ let

$$
L_{n} P(A):=\{f \in M(A)|\forall T \subseteq A,| T \mid \leqslant n \exists p \in P(A): f / T=p / T\}
$$

(These functions can be "interpolated" by polynomial functions on any $\boldsymbol{n}$ places.)

(e) $L P(A):=\cap_{n \in \mathrm{N}} L_{n} P(A)$. The elements in $L P(A)$ are the "local polynomial functions".

It is interesting to find all algebras which enjoy one of the following four properties:

Definition 2.3. An algebra $A$ is

polynomially complete if and only if $P(A)=M(A)$;

locally polynomially complete if and only if $L P(A)=M(A)$;

affine complete if and only if $P(A)=C(A)$;

locally affine complete if and only if $L P(A)=C(A)$.

Remarks 2.4.

(a) Our definitions in 2.3 differ slightly from those in (3)-(9), for these authors conisider also functions in several variables.

(b) One has (see e.g. (3))

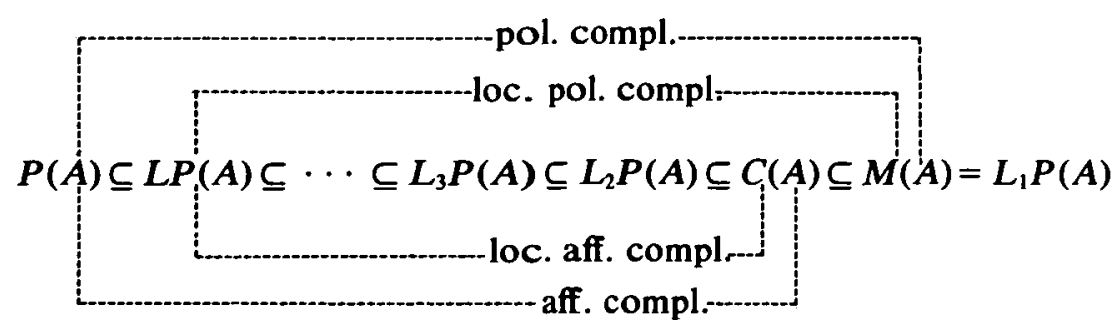

(c) If $\boldsymbol{A}$ is an $\boldsymbol{\Omega}$-group (the underlying group written additively) then all guys from $P(A)$ to $M(A)$ are near-rings w.r.t. + and o.

In this case, $C(A)$ can be written as

$$
C(A)=\{f \in M(A) \mid \forall I \& A \forall i \in I \forall a \in A: f(a+i)-f(a) \in I\}
$$

Hence all near-rings $N$ contained in $C(A)$ are gentle on $A$ if $A$ is just a group (or, more generally, if every normal subgroup of $(A,+)$ is an ideal of $A$, as is the case for the rings $Z$ and $Z_{n}$ ).

(d) Every polynomially complete algebra is simple (see e.g. (6)).

\section{Examples 2.5.}

(a) Let $A$ be a commutative ring with identity. Then the polynmial functions are as usual.

$A$ is polynomially complete iff $A$ is a finite field (see (6)).

By Lagrange's theorem, $R$ is locally polynomially complete. 
So $P\left(Z_{2}\right)=M\left(Z_{2}\right)$, but (see (14))

$P\left(\mathrm{Z}_{4}\right)=C\left(\mathrm{Z}_{4}\right)$, and

$$
P\left(Z_{8}\right)=\left\{\begin{array}{l|l}
f \in C\left(Z_{8}\right) & \begin{array}{l}
f(4)=2 f(2)-f(0), f(6)=f(3)+f(4)-f(0) \\
f(5)=2 f(3)-f(1), f(7)=6 f(1)+3 f(2)
\end{array}
\end{array}\right\}
$$

For further results, see (14) and (7).

(b) Let $(A,+)$ be a group.

Then $P(A)=\left\{f: a \rightarrow a_{0}+n_{1} a+a_{1}+\cdots+n_{r} a+a_{r} \mid r \in N_{0}, a_{i} \in A, n_{i} \in \mathbb{Z}\right\}$

$$
=I(A)+M_{c}(A) \quad((6) \text { and }(7))
$$

(where $I(A)$ is the d.g. near-ring generated by the inner automorphisms of $A$ ).

The polynomially complete groups are $Z_{2}$ and the finite non-abelian simple groups, (6). For more, see (7), (9), (3) and (4).

Now we try to apply the structure theory of near-rings to get further results. In order to do this, we need some lemmas.

Lemma $2.6(9,12)$. Let the universal algebra $A$ be a subdirect product of the algebras $A_{i}(i \in I)$. Then for all $f \in C(A)$ there exist uniquely determined $f_{i} \in C\left(A_{i}\right)$ with $f\left(\left(\ldots, a_{i}, \ldots\right)\right)=\left(\ldots, f_{i}\left(a_{i}\right), \ldots\right)$ for all $\left(\ldots, a_{i}, \ldots\right) \in A$. If $f \in P(A)$ then all $f_{i}$ are in $P\left(A_{i}\right)$.

From now on, we will exclusively deal with $\Omega$-groups, the group operation written additively with neutral element 0 . First we need some converse of 2.6 .

Notation 2.7. (a) Let $P_{0}(A)$ and $C_{0}(A)$ be the zero-symmetric parts of $P(A)(C(A)$, respectively), i.e. the subnear-rings consisting of all maps which take 0 into 0 .

(b) Let $A$ be the direct sum of the $\Omega$-groups $A_{i}(i \in I)$ and let $f_{i}$ be in $C\left(A_{i}\right)$ such that almost all (i.e. all but a finite number) of the $f_{i}$ 's belong to $C_{0}\left(A_{i}\right)$. Define $f:=\oplus_{i \in I} f_{i}$ by $f\left(\left(\ldots, a_{i}, \ldots\right)\right):=\left(\ldots, f_{i}\left(a_{i}\right), \ldots\right)$ for $\left(\ldots, a_{i}, \ldots\right) \in A$.

Definition 2.8. Let the situation be as in 2.7(b).

(a) The direct sum of the $A_{i}$ 's is nice if $f \in C(A)$.

(b) The direct sum of the $A_{i}$ 's is kind if $f \in P(A)$ whenever all $f_{i}$ are in $P\left(A_{i}\right)$.

Proposition 2.8 (cf. (9)). Let $A$ be the direct sum of the $\Omega$-groups $A_{i}(i \in I)$, such that $A$ has no "skew congruences", which means that all ideals of $A$ are direct sums of ideals of $A_{i}$. Then this sum is nice.

Proof. Take $f_{i} \in C\left(A_{i}\right)$ such that almost all $f_{i}$ are in $C_{0}\left(A_{i}\right)$. Then $\oplus_{i \in I} f_{i}: A \rightarrow A$. We have to show that $f \in C(A)$. Take $J \leqslant A$ and $\left(\ldots, a_{i}, \ldots\right),\left(\ldots, b_{i}, \ldots\right) \in A$ such that $\left(\ldots, a_{i}, \ldots\right)-\left(\ldots, b_{i}, \ldots\right)$ is in $J$. Then $J$ splits into the direct sum $\oplus_{i \in I} J_{i}$ with $J_{i} \leqslant A_{i}$. Hence all $a_{i}-b_{i} \in J_{i}$. Since all $f_{i}$ are compatible, we get $f\left(\left(\ldots, a_{i}, \ldots\right)\right)-$ $f\left(\left(\ldots, b_{i}, \ldots\right)\right)=\left(\ldots, f_{i}\left(a_{i}\right)-f_{i}\left(b_{i}\right), \ldots\right) \in \bigoplus_{i \in I} J_{i}=J$ and $f$ is shown to be compatible.

Examples 2.9. It is shown in (15) that direct sums of rings with identity are kind; it is mentioned in (9) that (finite) direct sums of rings with identity or of finite groups with relatively prime order are nice. 
This motivated the following

Conjecture 2.10. A direct sum is nice iff it is kind, which happens iff $A$ has no skew congruences.

Definition 2.11. For an $\Omega$-group $A$ let

$$
\begin{gathered}
L_{2,5} P(A):=\left\{f \in M(A) \mid \forall a_{1}, a_{2} \in A \exists p \in P(A): \quad p(0)=f(0), p\left(a_{1}\right)=f\left(a_{1}\right),\right. \\
\left.p\left(a_{2}\right)=f\left(a_{2}\right)\right\} .
\end{gathered}
$$

Remark 2.12. $L_{2,5} P(A)$ is then a near-ring between $L_{3} P(A)$ and $L_{2} P(A)$. (It is known that for $\Omega$-groups $L_{2} P(A)=C(A)$.)

From the density theorem for near-rings (see e.g. (10)) we get that "2,5-fold transitivity implies density":

Theorem 2.13. Let $A$ be an $\Omega$-group such that $P_{0}(A)$ is no ring. If $M(A)=$ $L_{2,5} P(A)$ then $A$ is locally polynomially complete.

Proof. If $A$ is as in the statement then $N:=P(A)$ is as follows: $N_{0}$ is not a ring, $N \neq N_{0}$ is 2-fold transitive on $A-\{0\}$. By Theorem 4.65 of (10), $N$ is dense in $M(A)$, thereby fulfilling the finite interpolation property, whence $M(A)=L P(A)$.

Examples 2.14. (a) Let $A$ be a group. Then $P_{0}(A)$ is a ring iff $A$ is an "L-group", which means that all conjugated elements commute (see (2) or (10)).

(b) If $A$ is a ring with identity then $P_{0}(A)$ is a ring iff $A$ is a Boolean ring. If $A$ is a simple ring then $P_{0}(A)$ is a ring iff $A$ is either isomorphic to the field $Z_{2}$ or if $A$ is a zeroring (see (12)).

The next proposition holds for all $n \in N$ instead of 2,5 , but we will need it only in this case. $C(B)$.

Proposition 2.15. Let $A=B \oplus C$ be nice. If $L_{2,5} P(A)=C(A)$ then $L_{2.5} P(B)=$

Proof. Take $b_{1}, b_{2} \in B$ and some $f \in C(B)$. Define $\bar{f}: A \rightarrow A$ by $\bar{f}(b+c):=f(b)$. Then $\bar{f}$ is the direct sum of $f$ and the zero map in the sense of $2.7(\mathrm{~b})$, and hence compatible. Therefore $\exists p \in P(A): p(0)=\bar{f}(0), p\left(b_{i}\right)=\bar{f}\left(b_{i}\right)(i=1,2)$. If $p$ splits into $p_{A} \oplus p_{B}$ as in 2.6 then $p_{A}$ "interpolates" $f$ at 0 and at $b_{1}, b_{2}$. Therefore $L_{2,5} P(B)=$ $C(B)$.

Corollary 2.16. Let $A$ be a nice direct sum of $\Omega$-groups $A_{i}(i \in I)$. If $L_{2,5} P(A)=$ $C(A)$ then $L_{2,5} P\left(A_{i}\right)=C\left(A_{i}\right)$ for all $i \in I$.

Theorem 2.17. If $A$ is a kind direct sum of simple $\Omega$-groups $A_{i}(i \in I)$ such that no $P_{0}\left(A_{i}\right)$ is a ring and $L_{2,5} P(A)=C(A)$ then $A$ is locally affine complete.

Proof. If $i \in I$ then, by $2.16, L_{2.5} P\left(A_{i}\right)=C\left(A_{i}\right)=M\left(A_{i}\right)$ (since $A_{i}$ is simple), so 
$\operatorname{LP}\left(A_{i}\right)=M\left(A_{i}\right)$ by 2.13. Now take $a_{1}=\left(\ldots, a_{1 i}, \ldots\right), \ldots, a_{n}=\left(\ldots, a_{n i}, \ldots\right) \in A$ and some $f \in C(A)$ which may split into $\bigoplus_{i \in I} f_{i}$ as in 2.6. Then

$$
\forall i \in I \exists p_{i} \in P\left(A_{i}\right) \forall k \in\{1, \ldots, n\}: p_{i}\left(a_{k i}\right)=f_{i}\left(a_{k i}\right) \text {. }
$$

Almost all $p_{i}$ can be taken out of $P_{0}\left(A_{i}\right) \subseteq C_{0}\left(A_{i}\right)$. If $p:=\oplus_{i \in I} p_{i}$ then $p \in P(A)$ and $\forall k \in\{1, \ldots, n\}: p\left(a_{k}\right)=f\left(a_{k}\right)$. So $L P(A)=C(A)$ and $A$ is locally affine complete.

Corollary 2.18. Let $A$ be the direct sum of simple rings with identity and let $L_{2,5} P(A)=C(A)$. Then $A$ is locally affine complete.

Proof. By 2.9, direct sums of rings with identity are kind. $P_{0}\left(A_{i}\right)$ is no ring unless $A_{i}$ is a simple Boolean ring; in this case, $A_{i}$ is isomorphic to $Z_{2}$. But then we also get that $L P\left(A_{i}\right)=P\left(A_{i}\right)=C\left(A_{i}\right)=M\left(A_{i}\right)$ which shows that in this case 2.17 also works if $P_{0}\left(A_{i}\right)$ is a ring.

Problems 2.19. Is 2.10 true?

Since simple non-abelian groups are not $L$-groups, it makes sense to ask: Let $A$ be the direct sum of simple non-abelian groups. Does $L_{2,5} P(A)=C(A)$ imply that $A$ is locally affine complete?

When is the direct sum of polynomially complete (locally polynomially complete) groups, rings, etc. locally affine complete?

Can one get similar results concerning chain conditions for gentle near-rings as for tame near-rings?

\section{Structure theorems}

Again, let $A$ be an $\Omega$-group, written additively with zero element 0 . Polynomials are excellent for describing generated ideals:

Theorem 3.1. For $a \in A$, let $\langle a\rangle$ be the principal ideal of $A$ generated by $a$. Then $\langle a\rangle=\left\{p(a) \mid p \in P_{0}(A)\right\}($ see 2.8).

Proof. (a) Let $N:=P_{0}(A)$. Since $\langle a\rangle \leqslant A,\langle a\rangle \leqslant{ }_{N} A$ by 2.4 (c), hence $\langle a\rangle{ }_{N} A$, whence $\left\{p(a) \mid p \in P_{0}(A)\right\}=N a \subseteq\langle a\rangle$.

(b) Conversely, we will show that this $\mathrm{Na}$ is an ideal of $A$ containing $a$, from which we get $\langle a\rangle \subseteq N a$.

(i) Because of $\operatorname{id}_{A} \in N, a=\operatorname{id}(a) \in N a$.

(ii) Clearly, $\mathrm{Na}$ is a subgroup of $(A,+)$.

(iii) $\forall b \in A \forall p(a) \in N a: b+p(a)-b=(\underline{b}+p-\underline{b})(a)$, where $\underline{b}$ is the constant polynomial function with value $b$. Now $\underline{b}+p-\underline{b} \in P(A)$ and $(\underline{b}+p-\underline{b})(0)=$ $b+p(0)-b=b-b=0$, so $\underline{b}+p-\underline{b} \in N$ and $b+p(a)-b \in N a$.

Hence $\mathrm{Na}$ is normal in $(A,+)$.

(iv) Let $\omega$ be an $n$-ary operation on $A$ and $b_{1}, \ldots, b_{n} \in A, p_{1}(a), \ldots, p_{n}(a) \in N a$. Consider

$$
b:=\omega\left(b_{1}+p_{1}(a), \ldots, b_{n}+p_{n}(a)\right)-\omega\left(b_{1}, \ldots, b_{n}\right) .
$$


Let $q$ be the polynomial $\omega\left(\underline{b}_{1}+p_{1}, \ldots, \underline{b}_{n}+p_{n}\right)-\omega\left(\underline{b}_{1}, \ldots, \underline{b}_{n}\right)$. Then $q(a)=b$ and $q(0)=0$, therefore $b=q(a) \in N a$.

Theorem 3.2. Let $N$ be a near-ring between $P_{0}(A)$ and $C(A)$. Then every minimal ideal $S$ of $A$ is an $N_{0}$-group of type 2.

Proof. (i) Since $S \leqslant A, S \mho_{N} A$ by $2.4\left(\right.$ c), so $S \leqslant{ }_{N_{0}} A$ which shows that $S$ is an $N_{0}$-group.

(ii) Because of $\mathrm{id}_{A} \in N_{0}$ we get $N_{0} S=S \neq\{0\}$.

(iii) By 3.1, the ideal $\langle s\rangle$ generated by $s \in S$ is given by $\left\{p(s) \mid p \in P_{0}(A)\right\}$.

(iv) Let $T \leqslant{ }_{N_{0}} S$. Then $\forall n \in N_{0} \forall t \in T: n(t) \in T$. By (iii), $\forall t \in T:\langle t\rangle \subseteq T$, so $T \leqslant$ $A$ and $T \subseteq S$.

(v) Since $S$ is minimal, we see from (iv) that $S$ is $N_{0}$-simple.

(vi) Let $s_{0}$ be in $S-\{0\}$. Then $N_{0} s_{0}=\left\langle s_{0}\right\rangle=S$. So ${ }_{N_{0}} S$ is strictly monogenic and of type 2.

From (iv) of the preceding proof we see that every $N_{0}$-subgroup of $A$ is an ideal of $A$, hence an ideal of ${ }_{N} A$. So we get (on $A$ ).

Corollary 3.3. Every zero-symmetric near-ring between $P_{0}(A)$ and $C(A)$ is tame

So in this case, "N-subgroup", "ideal of ${ }_{N} A$ " and "ideal of $A$ " all mean the same.

\section{Theorem 3.4.}

(a) Let $A$ in 3.2 be a simple group. Then $P(A)$ is dense in $M_{\text {aff }}(\Gamma)$ (if $A$ is abelian) or otherwise in $M(A)$.

(b) If $A$ is a simple ring with identity then $P(A)$ is dense in $M(A)$.

In the second case of (a) and in (b), $A$ is locally polynomially complete (5).

Proof. (a) follows from the density theorem 4.52 of $(10)$ and the fact that $P_{0}(A)$ is a ring iff $A$ is an $L$-group; but a simple $L$-group is abelian. $P(A)$ is 2 -primitive on $A$.

We only have to show that $G:=\operatorname{Aut}_{P_{0}(A)}(A)=\{\mathrm{id}\}(10,4.54)$ if $A$ is non-abelian:

Let $h \in G$. Suppose that $h \neq$ id. Then $\exists a \in A:-a+h(a) \notin\{0\}=Z(A,+)$ (the centre of $A)$; so $\exists b \in A:-a+h(a)+b \neq b+(-a+h(a))$.

Since $h$ is $1-1, \exists c \in A: b=h(c)$. a.

$$
\text { Hence }(-a+h(a))+h(c)-(-a+h(a)) \neq h(c) \text {, so } h(a)+h(c)-h(a) \neq a+h(c)-
$$

Take $p_{0}: A \rightarrow A: x \rightarrow a+x-a$.

Then $p_{0} \in P_{0}(A)$.

$$
\begin{aligned}
h(a)+h(c)-h(a) & =h\left(p_{0}(c)\right) \text { and } \\
a+h(c)-a & =p_{0}(h(c)),
\end{aligned}
$$

whence $h \notin$ Aut $_{P_{\alpha}(A)}(A)$, a contradiction. (5).

In this second case, $A$ is locally polynomially complete-a result due to $H$. Kaiser 
(b) Let $A$ be a field.

$P_{0}(A)$ is a ring iff $A$ is Boolean (by 12).

If $A$ is Boolean then $A \cong Z_{2}$ and $M_{\text {aff }}\left(Z_{2}\right)=M\left(Z_{2}\right)$.

Anyhow, we compute (cf. (11) $\operatorname{Aut}_{P_{0}(A)}(A,+)$; in order to do this, we take some $h$ from it. Let $a \in A \backslash\{0\}$.

Since $h\left(x^{2} \circ a\right)=x^{2} \circ h(a), h\left(a^{2}\right)=h(a)^{2}$.

Also, $h(a x \circ a)=a x \circ h(a)$, so $h\left(a^{2}\right)=a h(a)$.

Hence $(a-h(a))(h(a))=0$. Now take $a:=h^{-1}(1)$. Then $(a-1) \cdot 1=0$, whence $1=a=h^{-1}(1)$. Since Aut $P_{P_{0}(A)}(A)$ is fixed-point-free by (1) or $(10), h^{-1}=h=\mathrm{id}$.

By the density theorem, $P(A)$ is dense in $M(A)$.

So in any case, $P(A)$ is dense in $M(A)$ and $A$ is locally polynomially complete.

Theorem 3.5. Let $A$ either be a group or a ring with identity and the direct sum of simple (or minimal) $A_{i}$ 's of the same kind.

Then $P(A)$ is a subdirect product of 2-primitive near-rings $N_{i}=C\left(A_{i}\right)(i \in I)$ which are dense in

(a) $M_{\mathrm{aff}}\left(A_{i}\right)$ (where $D_{i}$ is a skew-field which makes $A_{i}$ into a vector space) iff $A_{i}$ is an abelian group

or in

( $\beta) M\left(A_{i}\right)$ iff $A_{i}$ is a non-abelian group.

In the ring case, we always arrive at $(\beta)$.

Proof. (i) Since $A$ is completely reducible, the $A_{i}$ 's are minimal iff they are simple.

By 3.2, the $A_{i}$ 's are $P_{0}(A)$-groups of type 2 .

(ii) The annihilators $\left(0: A_{i}\right)$ are primitive ideals of $P_{0}(A)$ and $N_{i}:=P_{0}(A) /\left(0: A_{i}\right)$ is 2-primitive on $\left(A_{i},+\right)$ for all $i \in I$ with $\left(p+\left(0: A_{i}\right)\right)\left(a_{i}\right)=p\left(a_{i}\right)+\left(0: A_{i}\right)$ for all $p \in P_{0}(A)$ and $a_{i} \in A_{i}$ (see (10), 4.3b, 4.2b, and 3.14a). identity.

(iii) As a homomorphic image of $P_{0}(A), N_{i}$ is a zerosymmetric near-ring with

(iv) We consider the decomposition of $f \in P_{0}(A)$ into a family $\left(f_{i}\right)_{i \in I}$ according to 2.6. Then the map $\pi_{i}: P_{0}(A) \rightarrow P_{0}\left(A_{i}\right): f \rightarrow f_{i}$ is a homomorphism with kernel $\{g \in$ $\left.P_{0}(A) \mid \forall j \neq i: f_{i}=\emptyset\right\}=\left(0: A_{i}\right)$.

So $N_{i}=P_{0}(A) /\left(0: A_{i}\right) \cong \operatorname{Im} \pi_{i} \subseteq P_{0}\left(A_{i}\right)$ and we can replace the $N_{i}$ 's (if necessary) by their isomorphic copies $\operatorname{Im} \pi_{i}$ to get them into $P_{0}\left(A_{i}\right)$.

(v) In the case of rings with identity we know from 2.9 that $\operatorname{Im} \pi_{i}=P_{0}\left(A_{i}\right)$. So $N_{i}$ is dense in $M_{0}\left(A_{i}\right)$ by $3.4(b)$. For groups, we seemingly have no guarantee for equality. So we still have to work out the group case.

(vi) We have to ask: when is $N_{i}$ a ring?

If $A_{i}$ is abelian, $P_{0}\left(A_{i}\right)$ is a ring, and so is $\operatorname{Im} \pi_{i}$, hence also $N_{i}$.

Conversely; if $N_{i}$ is a ring then $\forall f \in P_{0}(A): f / A_{i} \in \operatorname{Hom}\left(A_{i}, A_{i}\right)$. In particular, we take $f: a \rightarrow a+a=2 a$. $f \in P_{0}(A)$, and for all $c, d \in A_{i}$ we get $c+d+c+d=f(c+d)=$ $f(c)+f(d)=c+c+d+d$, whence $d+c=c+d$ and $A_{i}$ is shown to be abelian. 
Hence $N_{i}$ is a ring iff $A_{i}$ is abelian.

(vii) If $A_{i}$ is non abelian then one sees as in the proof of 3.4(a) that $\operatorname{Aut}_{N_{i}}\left(A_{i}\right)$ is $=\{$ id $\}$ (the $p_{0}$ in this proof appears in $\operatorname{Im} \pi_{i}$ !).

(viii) We can now apply the density theorem 4.52 in (10) (due to G. Betsch (1)) to get $N_{i}$ dense in $\operatorname{Hom}_{D_{i}}\left(A_{i}, A_{i}\right)$ iff $A_{i}$ is abelian or dense in $M_{0}\left(A_{i}\right)$ in the other case.

(ix) Since $\cap_{i \in I}\left(0: A_{i}\right)=\{0\}$ by $2.6, P_{0}(A)$ is isomorphic to the subdirect product of the near-rings $N_{i}$.

(x) Finally, we can add the constant polynomials in $P(A)$, which can be decomposed into constant polynomials in $P\left(A_{i}\right)$ by 2.6 . In this way we get Hom $_{D_{i}}\left(A_{i}, A_{i}\right)+M_{c}\left(A_{i}\right)=M_{\text {aff }}\left(A_{i}\right)$ and $M_{0}\left(A_{i}\right)+M_{c}\left(A_{i}\right)=M\left(A_{i}\right)$, respectively.

Corollary 3.6. Let $A$ be as in 3.5. If $A$ is finite or, more generally, if $P_{0}(A)$ has the descending chain condition for left ideals, then $P(A)$ is isomorphic to a finite direct sum of $M_{\mathrm{aff}}\left(A_{i}\right)$ 's (where $A_{i}$ is a finite dimensional vector space) or $M_{0}\left(A_{i}\right)$ 's (according to the cases in 3.5).

Proof. If $P_{0}(A)$ fulfills the descending chain condition for left ideals then the same applies to all $N_{i}$ 's of the last proof. Hence each $N_{i}$ is isomorphic to $\operatorname{Hom}_{D_{i}}\left(A_{i}, A_{i}\right)$ or to $M_{0}\left(A_{i}\right)$, both being simple near-rings. Hence all $\left(0: A_{i}\right)$ turn out to be maximal, and the index set can be chosen to be finite by $2.52(\mathrm{~b})$ of (10).

Finally, we again add the constant maps as in the preceding proof.

Corollary 3.7. Let $A$ be as in 3.5. Then $P_{0}(A)$ is 2-semisimple.

Problems 3.8. Which classes of algebras have the property that direct sums of (locally) polynomially complete algebras are locally affine complete?

Can one conclude some kinds of completeness out of certain subdirect representations?

When is $S$ in 3.2 faithful (then $N_{0}$ would be 2-primitive on $S$ with identity, so the density theorem is at hand)?

Acknowledgement. The author thanks Dr. Kaiser (Vienna) for a large number of highly appreciated suggestions and remarks.

Addendum. Private communications from Dr. Kaiser (Vienna, Austria) and Dr. Kaarli (Tartu, USSR) and the paper "On on the concept of length in the sense of Lausch-Nöbauer” by G. Eigenthaler (J. Austr. Math. Soc. 24 (1977), 162-169) have revealed the answer to Conjecture 2.10:

$A$ is nice $\Leftrightarrow A$ has no skew congruences $\Leftarrow A$ is kind. $S_{3} \oplus Z_{3}$ is nice, but not kind. For abelian groups, "nice" and "kind" are equivalent. See also the forthcoming paper "On near-rings generated by the endomorphisms of some groups" by K. Kaarli. This paper implies that $L_{2,5} P(A)=C(A) \Rightarrow A$ is locally affine complete (2.19).

\section{REFERENCES}

(1) G. BeTSCH, Primitive near-rings, Math. Z. 130 (1973), 351-361.

(2) A. J. Chandy, Rings generated by inner automorphisms of non-abelian groups, Proc. Amer. Math. Soc. 30 (1971), 59-60. 
(3) H. HUle and W. NÖBAUER, Local polynomial functions on universal algebras, submitted.

(4) H. HULE and W. NÖBAUER, Local polynomial functions on abelian groups, submitted.

(5) H. KAISER, Über lokal polynomvollständige universale Algebren, Abh. Math. Sem. Univ. Hamburg 43 (1975), 158-165. 1973).

(6) H. LAUSCH and W. NÖBAUER, Algebra of Polynomials (North-Holland, Amsterdam

(7) H. LAUSCH and W. NöBAuER, Funktionen auf endlichen Gruppen, Publ. Math. Univ. Debrecen 23 (1976), 53-61.

(8) W. NÖBAUER, Compatible and conservative functions on residue-class rings of the integers, Coll. Math. Soc. János Bolyai 13 (Topics in Number Theory, 1974), 245-257.

(9) W. NÖBAUER, Über die affin vollständigen, endlich erzeugbaren Moduln, Monatsh. Math. 82 (1976), 187-198.

(10) G. PILZ, Near-rings (North-Holland, Amsterdam, 1977).

(11) G. PILz, Quasi-anelli: teoria ed applicazioni, Rendic. Mat. (Milano), to appear.

(12) G. PILz and Y. S. So, Near-rings of polynomials and polynomial functions, J. Austral. Math. Soc., (to appear).

(13) S. P. SCOTT, Near-rings and near-ring modules (Ph.D. dissertation, Australian National Univ., 1970).

(14) Y. S. So, Polynom-Fastringe (Dissertation, Univ. Linz (Austria), 1978).

(15) H. WERNER, Produkte von Kongruenzklassengeometrien universeller Algebren, Math. Z. 121 (1971), 111-140.

JOHANNES KEPLER UNIVERSITÄT

LINZ

AUSTRIA 